九州大学学術情報リポジトリ

Kyushu University Institutional Repository

\title{
Propagation Mode Retention Using Strongly Coupled Multi-Core Fiber
}

Nasef, Mahmoud

Interdisciplinary Graduate school of Engineering Sciences, Kyushu University

Fuj imoto, Kantaro

Interdisciplinary Graduate school of Engineering Sciences, Kyushu University

Jiang, Haisong

Interdisciplinary Graduate school of Engineering Sciences, Kyushu University

Hamamoto, Ki ichi

Interdisciplinary Graduate school of Engineering Sciences, Kyushu University

https://doi.org/10.15017/2552944

出版情報: Proceedings of International Exchange and Innovation Conference on Engineering \& Sciences (IEICES). 5, pp. 81-83，2019-10-24. 九州大学大学院総合理工学府

バージョン:

権利関係 : 


\title{
Propagation Mode Retention Using Strongly Coupled Multi-Core Fiber
}

\author{
Mahmoud Nasef $^{1 *}$, Kantaro Fujimoto ${ }^{1}$, Haisong Jiang ${ }^{1}$, Kiichi Hamamoto ${ }^{1}$ \\ ${ }^{1}$ Interdisciplinary Graduate school of Engineering Sciences, Kyushu University, Japan \\ *Corresponding author email: mmnasef@kyudai.jp
}

\begin{abstract}
This paper deals with the research proposal and evaluation of Strongly coupled MCF which is studied in propagation mode retaining capability as regular multi-mode fiber has an issue of mode crosstalk. As a result, mode retention characteristics of $5 \mathrm{~dB}$ at $80 \mathrm{~mm}$ bending radius has been confirmed successfully.
\end{abstract}

Keywords: Strongly coupled fiber; Mode crosstalk; Multi-core fiber; Mode division multiplexing.

\section{INTRODUCTION}

Data center traffic is already estimated to become $20 \mathrm{ZB}$ by the year 2021 about twice as much as in 2019 [1] MDM (mode division multiplexing) can expand transmission capacity within data center [2]; therefore, we propose to introduce an optical network system utilizing MDM into data centers [3]. It has; however, a problem of mode crosstalk in the fiber that prevents proper preservation of individual mode transmission. One main cause of this crosstalk has been mode excitation phenomenon at the bending region of the fiber [4]. MIMO (Multi-input multi-output) is exploited to demonstrate MDM transmission capability; however, it is ideal in the case of utilizing mode retaining that leads to MIMO less transmission. In this paper, we study and demonstrate strongly coupled MCF to verify the mode retaining capability. We simulated and estimated the transmittance of input mode by using BPM (beam propagation method) simulation. As a result, we verified the mode retention characteristics of $5 \mathrm{~dB}$ at $80 \mathrm{~mm}$ bending radius, compared to regular multi-mode fiber.

\section{CONCEPTS}

\subsection{Propagation Mode in Strongly Coupled MCF}

In MDM, LP (linear polarization) mode has mainly been researched in recent years; however, LP mode has some problems in applying it to transmission within data centers, especially, the existence of mode degeneration [5]. Thus, it normally requires the use of MIMO processing which is not suitable for data centers. One way to avoid the mode degeneration issue is to use single dimensional mode and strongly coupled MCF for the transmission line.

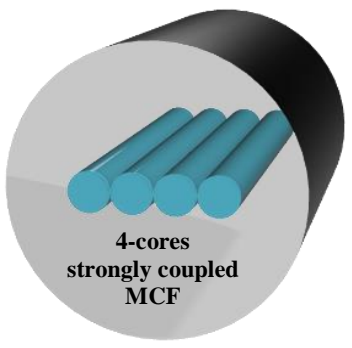

(a)

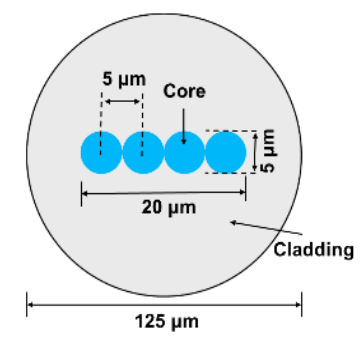

(b)
Fig. 1. Strongly coupled MCF which propagates strongly coupled beams. (a) Schematic; (b) Crosssectional structure view.

A schematic view of the fiber being used is shown in Fig.1. Here, the fiber used is a coupled multi-core fiber which consists of 4 single-mode cores strongly coupled together as shown in Fig.1 (a). This strongly coupled MCF propagates strongly coupled beams with each core width of $5 \mu \mathrm{m}$ as illustrated in Fig.1 (b). The fiber length used is about $4 \mathrm{~m}$ long. In the coupled MCF, cores with small diameter are adjacent in horizontal directions (the refractive indices of core and cladding are $n_{1}=1.45886$ and $\mathrm{n}_{2}=1.43824$, respectively) [6].

\subsection{Mode Crosstalk in Fiber}

Mode crosstalk may occur in the transmission line. This may be caused at the bending region of the fiber. This phenomenon is explained in Fig.2. This figure shows one example of the simulation by using beam propagation method (BPM). Higher mode may be excited especially in the case of small bending radius. This leads to mode crosstalk. In the case of Fig.2, $\mathrm{LP}_{01}$ mode is injected whereas $\mathrm{LP}_{11}$ mode is excited at the bending region [5]. Because of the mode crosstalk, MIMO is essential when we want to distinguish the modes; however, MIMO is not

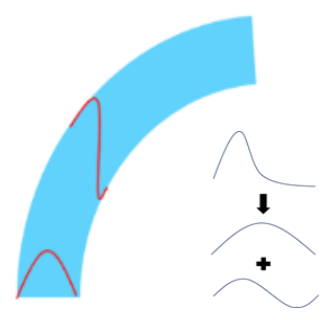

(a)

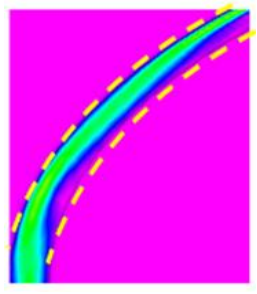

(b) desired in short distance transmissions such as within data centers due to the latency in addition to the cost issues.

Fig. 2. Light un-centered and higher mode excitation at bending region of regular multi-mode fiber. (a) Schematic; (b) Simulation. 


\subsection{Concept of Mode Retention}

The beam going outside at the bending region (fiber or waveguide) may be prevented in the strongly coupled MCF (multi-core fiber) as shown in Fig.3. The strongly coupled MCF may prevent higher order mode excitation at the bending region as the core spacing may prevent beam deflection toward bending outside.
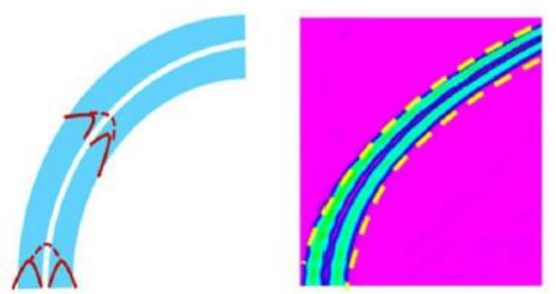

Fig. 3. Mode propagation at bending waveguide/region in proposing slit waveguide.

\section{RESULTS AND DISCUSSION}

\subsection{Simulation Results}

We estimated the $0^{\text {th }}$ mode transmittances of the strongly coupled MCF and regular MMF by BPM simulation. Input light used was $1550 \mathrm{~nm}$ TE $0^{\text {th }}$ mode. In order to verify mode retention characteristics in bending strongly coupled MCF, we estimated $0^{\text {th }}$ mode transmittance per one turn (custom reel) after calculating and standardizing the $0^{\text {th }}$ mode transmittance in 5 degree bending fiber. The overall results of four different bending radii are shown in Fig. 6. (a). In the case of strongly coupled MCF, the $0^{\text {th }}$ mode transmittance was improved to about $3 \mathrm{~dB}$ in 40 $\mathrm{mm}$ bending radius, so we confirmed mode retention characteristics of strongly coupled MCF. Note that the simulation was done for the parallel direction bending against the core-arrangement line as shown in Fig.4.

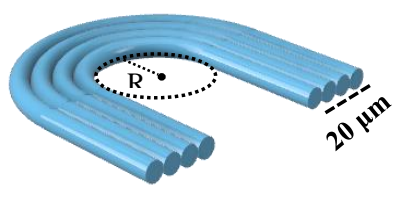

(a)

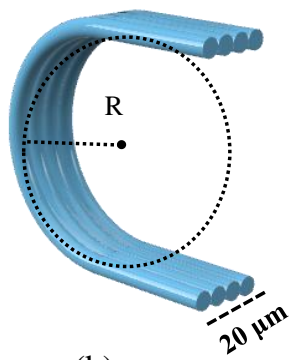

(b)
Fig. 4. Bending directions of the strongly coupled MCF against core-arrangement line. (a) Parallel arrangement, (b) Perpendicular arrangement.

\subsection{Experimental Results}

Firstly, Fig. 5 below illustrates the far field pattern (FFP) intensity measurement of $0^{\text {th }}$ mode and $1^{\text {st }}$ mode. We conducted an experimental setup that demonstrates the effect of bending radius of strongly coupled MCF and observed the findings. We could change the bending radius of the fiber by turning the fiber one turn using a custom made reel. In addition, four different bending radius measuring points $(50 \mathrm{~mm}, 80 \mathrm{~mm}, 110 \mathrm{~mm}, 150$ $\mathrm{mm}$ ) were considered in order to observe the changes in the propagation mode retention and suppression of any mode excitation.

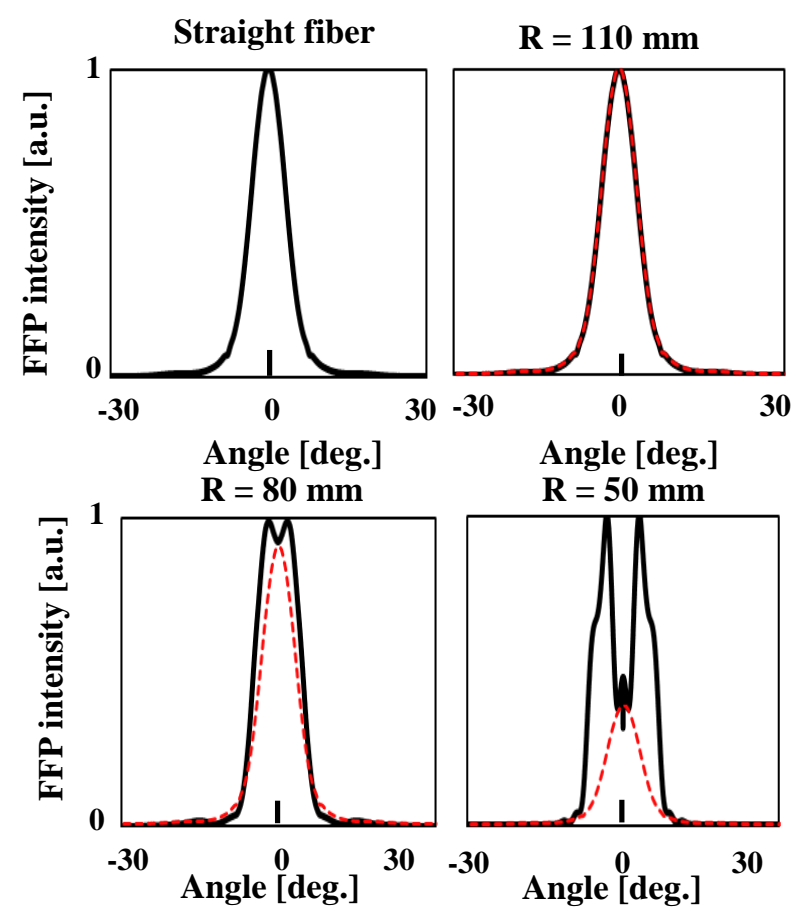

Fig. 5. FFP intensity measurement of strongly coupled $\mathrm{MCF}$ at different bending radii $(\mathrm{R})$.

We validated the FFP intensity profiles along the horizontal center axis and the results are shown in Fig. 5. Initially, by observing Fig. 6. (b) below, $0^{\text {th }}$ mode transmittance of the strongly coupled MCF is higher than those of the few mode fiber (FMF). When the bending radius of the fiber is at $80 \mathrm{~mm}$, mode retaining effect of $5 \mathrm{~dB}$ was confirmed. Furthermore, by comparing both results of Fig. 6. (a) and (b), both data show that strongly coupled MCF looks better in mode retention than the regular FMF when it comes to mode retention along the bending region.

However, the $0^{\text {th }}$ mode transmittance of the strongly coupled MCF of the experiment seems higher than that of the simulation results. The reason may be due to the different fiber bending directions. For the experimental work, the direction of the fiber could not be arranged to only parallel direction. Since mode retention is better in the perpendicular bending arrangement case in Fig.4 (b), the experiment resulted in better mode retaining egpability than that of the simulation.

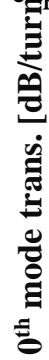

है
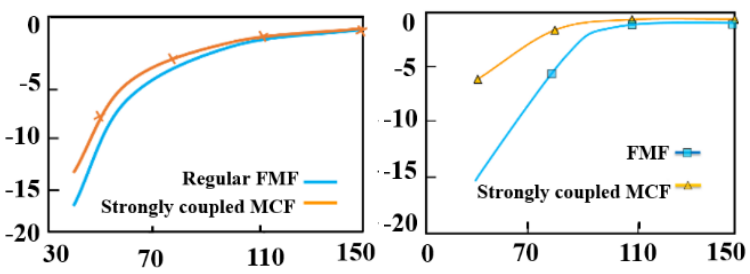

Bending radius [mm]

(a)

(b)

Fig. 6. (a) Simulation results, (b) Experimental results of $0^{\text {th }}$ mode transmittance and mode retaining effect by reeling the strongly coupled $\mathrm{MCF}$. 


\section{CONCLUSION}

By using strongly coupled MCF, the simulation data estimated that the $0^{\text {th }}$ mode transmittance is about $3 \mathrm{~dB}$ better at the bending radius of $40 \mathrm{~mm}$ and the mode retention effect may be higher as the bending radius is smaller for the regular multimode fiber.

Additionally, the experimental results verified and improved on the simulated work. We verified mode retention characteristics of $5 \mathrm{~dB}$ at $80 \mathrm{~mm}$ bending radius, compared to the regular multi-mode fiber. Therefore, the experimental measurement results suggested the propagation mode retention characteristics of strongly coupled MCF.

\section{ACKNOWLEDGMENT}

This work has been financially supported by JSPS and technical support with fiber implementation by Fujikura Ltd, are all gratefully acknowledged.

\section{REFERENCES}

[1] "Cisco Global Cloud Index: Forecast and Methodology, 2016-2021 White Paper" https://www.cisco.com/c/en/us/solutions/collateral/s ervice-provider/global-cloud-index-gci/white-paperc11-738085.html

[2] Y. Kokubun, T. Komo, K. Takenaga, S. Tanigawa and S. Matsuo, "Quantitative mode discrimination and bending crosstalk of four-core homogeneous coupled multi-core fiber," OFC/NFOEC, Los Angeles, CA, 2012, pp. 1-3.

[3] Y. Kokubun, M. Koshiba, "Novel multi-core fibers for mode division multiplexing: proposal and design principle". IEICE Electronics Express, 6(8), 2009, pp.522-528.

[4] T. Hayashi, T. Nagashima, O. Shimakawa, T. Sasaki and E. Sasaoka, "Crosstalk variation of multi-core fibre due to fibre bend," 36th European Conference and Exhibition on Optical Communication, Torino, 2010, pp. 1-3.

[5] K. Hamamoto, H. Jiang, "Optical mode switch and mode selective light source toward flexible modedivision multiplexing network." pp. PTh1D-4. Photonics in Switching. Optical Society of America, 2017.

[6] Snyder, A.W. and Love, J.D., 1986. Optical waveguide theory. J. Opt. Soc. Am. A, 3, p.378. 